\title{
Nailfold capillaroscopic changes in Kindler syndrome
}

\author{
Hristo P. Dobrev*, Nina I. Vutova \\ Department of Dermatology and Venereology, Medical University, Plovdiv, Bulgaria.
}

\begin{abstract}
Summary Kindler syndrome (KS), the fourth major type of hereditary epidermolysis bullosa (HEB), is a rare, autosomal recessive disorder characterized by skin fragility and blistering at birth followed by development of marked photosensitivity and progressive poikilodermatous skin changes in later years. We reported here the case of a 54-year-old woman, who fulfills the diagnostic criteria of KS type of $\mathrm{HEB}$, putting accent on the nailfold capillaroscopic changes. Using videocapillaroscopy we observed pronounced alterations in finger nail capillaries including reduction in capillary density, features of neoangiogenesis (architectural derangement, elongated loops, extremely tortuous, bushy or branching capillaries, thin, branching and interconnected capillaries), enlarged and giant capillaries. We consider the changes observed as an adaptive mechanism that compensate the loss of capillaries due to chronic periungual trauma. Further studies with larger number of patients are needed to confirm the significance of capillaroscopy findings for patients with HEB.
\end{abstract}

Keywords: Kindler syndrome, capillaroscopy

Kindler syndrome (KS), the fourth major type of hereditary epidermolysis bullosa (HEB), is a rare, autosomal recessive disorder characterized by skin fragility and blistering at birth followed by development of marked photosensitivity and progressive poikilodermatous skin changes in later years. After the first description by Theresa Kindler in 1954, more than 250 cases have been reported to date (1-4). In Bulgaria, about 100 patients suffered from HEB were currently registered. Among them only one case with KS is described (5). We report here a new case of KS putting accent on the nailfold capillaroscopic changes.

A 54-year-old woman presented with history of recurrent blistering after minor friction or trauma started after birth. The changes were more prominent on the extremities and tend to regress with age. Subsequently, photosensitivity, discoloration and atrophy of the skin developed. In addition, occasionally gingival and urethral bleeding, and surgically treated squamous cell carcinoma on the dorsum of the right hand 7 years ago were reported. The family history was negative. On examination, diffuse poikiloderma

*Address correspondence to:

Dr. Hristo P. Dobrev, Department of Dermatology and Venereology, Medical University, 15A V. Aprilov St., $4002-$ Plovdiv, Bulgaria.

E-mail: hristo_dobrev@hotmail.com (atrophy, telangiectases, and reticular pigmentation), mainly on the face and dorsal surfaces of the hands and feet, was observed. The dorsum of the hands and feet had atrophic skin with cigarette paper-like wrinkling. There were also skin erosions, atrophic scars, ectropion, gingivitis and periodontitis with missing teeth, and nail changes (prolonged eponychium, transverse and longitudinal ridges, onycholysis, yellow discoloration) (Figure 1). Routine blood tests, including immunological tests, were within normal range. The result from the nerve conduction study indicated the presence of sensory polyneuropathy. The histopathologic examination of skin lesion, performed 20 years ago, revealed an atrophic epidermis, subepidermal cracks, flattened dermis with thin collagen fibers, edema, dilated capillaries, pigmentary incontinence, and scarce perivascular infiltrate. A diagnosis KS type of HEB was made and symptomatic treatment was applied.

In order to explore the changes in microcirculation we performed nailfold videocapillaroscopy at varied magnifications $(\times 60, \times 200$, and $\times 500)$ using digital dermatoscope DinoLite (AnMo, Taipei, Taiwan). The following capillaroscopy findings were found: skin transparency: good; number: $4-6 / \mathrm{mm}$, reduction in capillary density; morphology: shape heterogeneity and marked tortuosity with varied appearance; dimensions: regularly and irregularly enlarged (width 30-50 


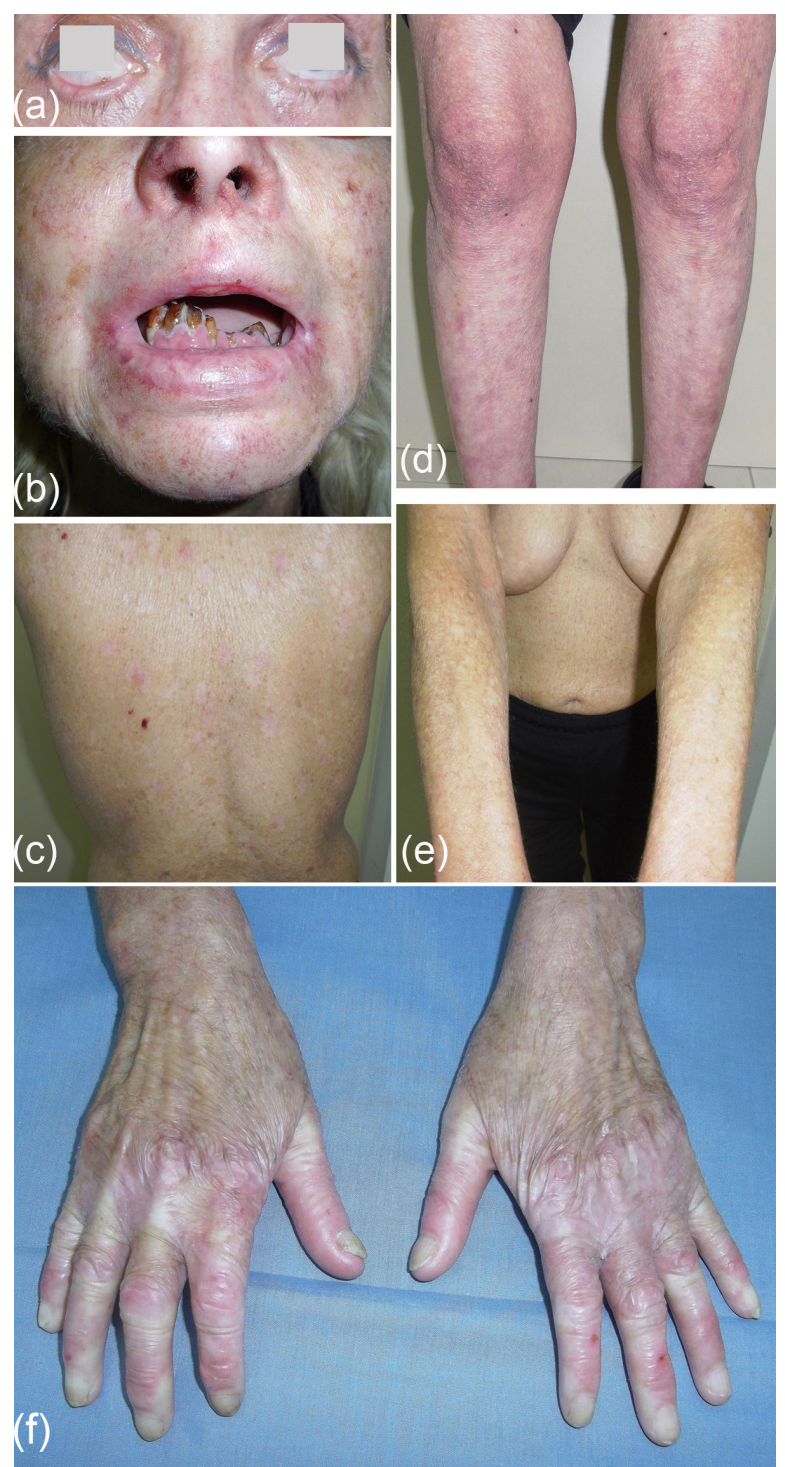

Figure 1. Ectropion (a); periodontitis (b); erythema, atrophy, hyper- and hypopigmented macules, teleangiectasia, xeroderma, atrophic scars (b-e), atrophic scarring with shiny cigarette paper-like wrinkling and small ulcerations on dorsum of the hands (f). micron) and giant capillaries (width $>50$ micron); distribution: architectural derangement and features of neoangiogenesis; blood flow: normal; absence of hemorrhages and thromboses. The interpretation was: presence of nailfold capillary microangiopathy (Figure 2).

$\mathrm{KS}$ is caused by a deficiency of the protein kindlin-1 due to a mutation in the gene KIND1 mapped to chromosome $20 \mathrm{p} 12$. It manifests during the neonatal period and infancy with skin fragility and trauma-induced skin blistering, which usually resolve with age. Later, varying degrees of photosensitivity and progressive poikiloderma develop. Face, hands and feet are most affected. Ocular and nail changes, periodontitis, esophageal, anal, vaginal and urethral stenosis have been also observed. Additionally, KS patients present an increased susceptibility for development of squamous cell carcinomas. The diagnosis is essentially clinical but could be supported by histopathologic examination, immunostaining with anti-kindlin-1 antibody, electron microscopy examination, and molecular genetic testing (1-4).

In 2005, Angelova-Fischer et al. (5) proposed the following diagnostic criteria for the syndrome: $i$ ) Major criteria: acral blistering in infancy and childhood, progressive poikiloderma, skin atrophy, abnormal photosensitivity, gingival fragility and/or swelling; ii) Minor criteria: syndactyly and mucosal involvement (anal, esophageal, urethral, laryngeal stenosis); iii) Associated findings: nail dystrophy, ectropion, palmoplantar keratoderma, leukoplakia, squamous cell carcinomas, skeletal abnormalities, periodontitis and tooth decay. The presence of 4 major criteria makes the diagnosis of KS certain. The presence of 3 major and 2 minor criteria makes the diagnosis probable and the presence of 2 major criteria and 2 minor criteria or associated symptoms renders the diagnosis likely. Our patient had all 5 major criteria, and mucosal and nail involvement, ectropion, squamous cell carcinoma, periodontitis and poorly preserved teeth.

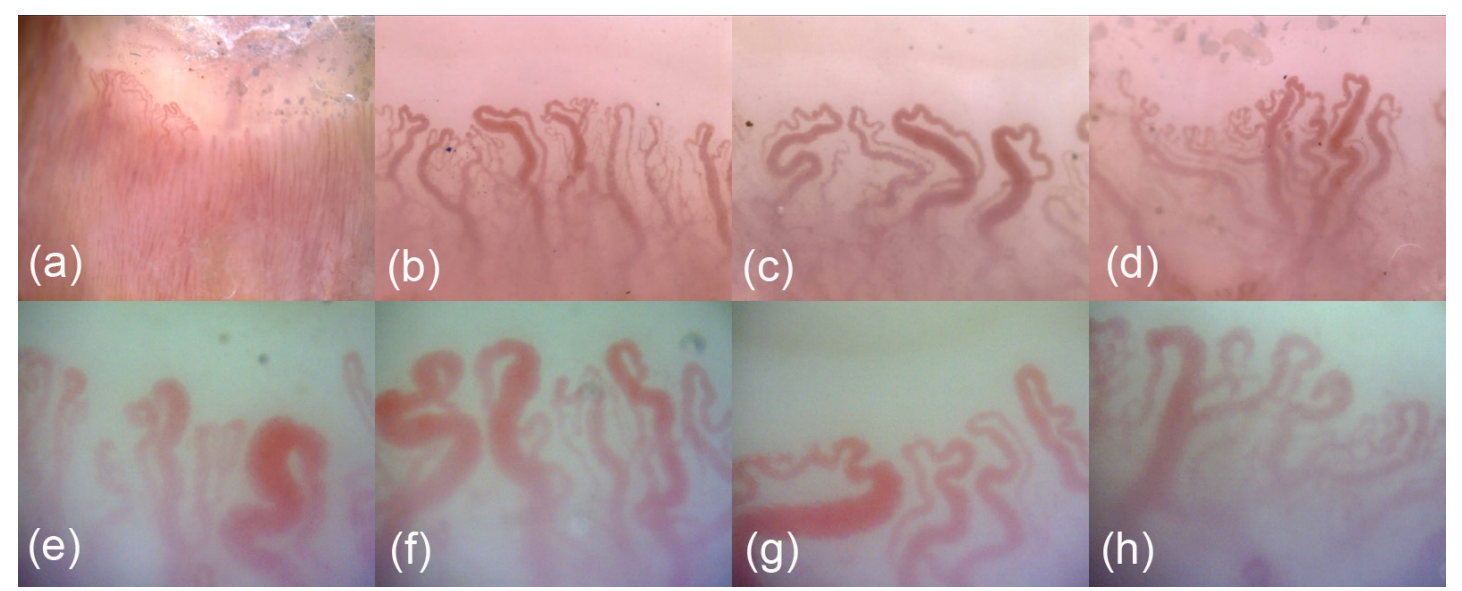

Figure 2. Capillaroscopic findings: reduction in capillary density, shape heterogeneity, marked tortuosity with varied appearance, enlarged and giant capillaries, architectural derangement, neoangiogenesis. Magnification: $\times 60$ (a); $\times 200$ (b-d); $\times 500(\mathbf{e}-\mathbf{h})$. 
So far there are not studies on microcirculation in HEB and KS in particular. That is why we aimed to evaluate the changes of nail fold microcirculation using non-invasive, digital videocapillaroscopy. We observed pronounced alterations in finger nail capillaries with the main feature of capillary neoformation including elongated loops, extremely tortuous, bushy or branching capillaries, thin, branching and interconnected capillaries. The presence of enlarged and giant capillaries makes the differentiation with Raynaud phenomenon somewhat difficult. Moreover, the clinical appearance of the patient's skin of the hands impress on general practitioners and rheumatologists that she suffer from autoimmune connective tissue disease. However, the patient did not report any vasospastic episodes of her fingers provoked by cold and the repeatedly immunological tests were normal. We consider the neoangiogenesis observed as an adaptive mechanism that compensate the loss of capillaries due to chronic periungual trauma. The role of sensory polyneuropathy could be additionally discussed. However, it is probably a consequence of the general disorder, too.

To the best of our knowledge, this is the first report of microcirculatory alterations in KS. Further studies with larger number of patients are needed to confirm the significance of capillaroscopy findings for patients with HEB.

\section{References}

1. Fine JD, Bruckner-Tuderman L, Eady RA, et al. Inherited epidermolysis bullosa: Updated recommendations on diagnosis and classification. J Am Acad Dermatol. 2014; 70:1103-1126.

2. Freiman A. (2013) Kindler Syndrome. http://emedicine. medscape.com/article/1118967-overview.htm1 (accessed September 30, 2015).

3. Zambruno G. (2013) Kindler syndrome. http://www.orpha. net/consor/cgi-bin/OC_Exp.php? lng $=$ EN\&Expert $=2908$ (accessed September 30, 2015).

4. Mendes L, Nogueira L, Vilasboas V. Talhari C, Talhari S, Santos M. Kindler syndrome: Report of two cases. An Bras Dermatol. 2012; 87:779-781.

5. Fischer IA, Kazandjieva J, Vassileva S, Dourmishev A. Kindler syndrome: A case report and proposal for clinical diagnostic criteria. Acta Dermatovenerol Alp Pannonica Adriat. 2005; 14:61-67.

(Received September 30, 2015; Revised October 17, 2015; Accepted October 19, 2015) 